\title{
ADEC-Energy Proficient Four-Level Deterministic Hierarchical Clustering Protocol for Wireless Sensor Network
}

\author{
Mamta Tewari ${ }^{1}$ and Kunwar Singh Vaisla ${ }^{2}$ \\ ${ }^{1}$ Department of Computer Science Engineering, BTKIT, Dwarahat-263656, \\ District- Almora, Uttarakhand, India \\ ${ }^{2}$ Department of Computer Science Engineering, BTKIT, Dwarahat, District- \\ Almora, Uttarakhand, India \\ mamta20tewari@gmail.com,vaislaks@ rediffmail.com
}

\begin{abstract}
As the popularity of Wireless sensor network is increasing and they are developed to perform a number of tasks, the issue of constrained energy of the sensor nodes is constantly raised by many researchers in past time. Many algorithms focus on the judicious use of the energy of sensor nodes to extend network lifetime for homogeneous as well as heterogeneous sensor networks. Clustering algorithms have been devised to optimize the energy consumption of a sensor network. In this paper we have extended the concept of heterogeneity in the deterministic energy efficient DEC protocol and proposed an advance deterministic energy proficient clustering technique for Wireless sensor network. We have implemented the DEC clustering algorithm in four level node hierarchies to enhance the sensor network life time, stability, and total remaining energy of the system. The simulated results show improved results in terms of energy consumption and hence in extending the network lifetime.
\end{abstract}

Keyword: WSN; clustering; ADEC; four level node hierarchy; energy efficient; DEC; network life time; FND, LND

\section{Introduction}

Wireless sensor network (WSN) has gain popularity [15] now-a-days due to its potential use in large number of areas. The WSN requirement was raised first in the applications related to military and now the use of WSN is extended to the field of environment monitoring, monitoring disaster hit areas; health care systems; surveillance systems. Research has been done in [2, 7, 9, and 10] exploring various latest applications of WSNs.

WSNs consist of hundreds or thousands of sensor node distributed arbitrary in a selected area $[7,15]$. The nodes circulate the sensed information to the base station or sink. The sensor nodes are energy constrained due to limited battery and are generally deployed for such applications where they cannot be accessed, so no possibility of replacing the battery. The energy constrained nature of WSNs makes it costly to employ in large scale. So over the years the prime focus of the researchers is to develop algorithms which can efficiently utilize the energy of sensor networks and can maximize lifetime of network. Clustering is a technique which aid in efficient processing of sensor network and extend the network lifetime [11]. Grouping sensor nodes within a cluster is profitable in terms of minimizing the energy consumption of nodes. In clustering, the nodes form a group known as cluster among them and choose a cluster head $(\mathrm{CH})$. The $\mathrm{CH}$ performs all the task of collecting and aggregating the sensed data and transmits it to the base station (BS) from cluster members [16]. Two types of networks, homogeneous and heterogeneous are formed due to clustering. In homogeneous system all the nodes are at same energy level while in the heterogeneous sensor network, there is some variation in 
the energy level of nodes. In past, numerous energy proficient protocols are proposed in $[1,4,6,8,12,13$, and 14]

In this paper, ADEC-energy proficient four-level deterministic hierarchical clustering technique is proposed with four-level node heterogeneity. This approach is an enhancement to the DEC [1] protocol which was developed for both homogeneous and heterogeneous system but in the heterogeneous system only three-level node hierarchy was considered. In the new proposed system the new node that is added to the system is called superior node with energy level greater than other nodes. The goal of new technique is to improve the performance of the DEC algorithm in increased heterogeneity. The cluster head $(\mathrm{CH})$ selection in ADEC depends on the concept of residual energy (RDE) [1] like in DEC. We have extended the three node hierarchy of DEC to four level node hierarchy systems. The setup and steady state phase of ADEC are same as in DEC. The main focus of the work is to further enhance the performance parameters of DEC algorithm with the inclusion of an extra node i.e., superior node. We have also analyzed our results against LEACH and DEC.

\section{Related Work}

In the literature of clustering algorithms various techniques are proposed to consider efficient energy utilization. LEACH (Low Adaptive Clustering Hierarchy) [4] was the first significant protocol proposed for energy optimization. In LEACH[4] the role of cluster head is rotated randomly and distribution of energy load is done equally among the sensor nodes. It is a probabilistic approach considers same energy level for all the nodes.

Authors introduced SEP [8] which is the first technique to discuss the concept of energy heterogeneity of nodes in WSNs. They have taken two types of nodes advance and normal nodes and the energy of advance node is greater than normal ones. Nodes are not mobile and are uniformly distributed over the sensing area.

HEED [12] is the clustering protocol which utilizes the residual energy as the key factor to select cluster heads and in case there is a tie between the nominee for cluster heads, network topology features are considered to break the tie. If a node falls within the territory of more than one cluster heads then a tie occurs.

D-SEP in [13] was proposed known as deterministic SEP is an improvement over SEP protocol. It works in rounds like SEP and focuses on optimal selection of cluster heads in heterogeneous environment. D-SEP increases the lifetime and stability of the network in heterogeneous network.

SEP-E in [14] extends SEP protocol taking three energy levels. The extended scheme improves networksstability region. In this technique new node called 'intermediate nodes' are added into the system, to provide the multi-node variety. It also improves the network lifetime

Authors in [6] proposed the DEEC protocol the cluster head is selected with the help of the initial and residual energy leveland these parameters also decide the duration of being cluster-heads. The odds of being the cluster head is more with the nodes with high initial and residual energy.

In [1], authors proposed deterministic energy efficient clustering protocol (DEC) for homogeneous as well as heterogeneous network in which the cluster head selection process is solely based on the concept of residual energy. It uses the network energy efficiently and gives better result as compared to the probabilistic techniques. In this technique a fixed number of cluster heads are chosen in each round thus improving the network lifetime and also removed the uncertainties involved in cluster head selection.

\section{Problem Statement}

Applications related to monitoring in WSN require a large number of nodes to be deployed which further leads to a huge number of data flow in the network. Due to this 
the energy consumption of the sensor network is high. In addition, data aggregation is also required in WSN as nodes residing nearby may sense and report same data. In past many algorithms are proposed but most of them considered the system to be homogeneous i.e energy level of all the nodes is same. Few algorithms in $[6,12,13$, and 14] brought the concept of heterogeneity to WSN. In [1] a deterministic clustering protocol is proposed which basically works in improving the stability of network with prolonging lifetime of network. It proves to be an improved technique than its predecessors such as SEP, LEACH and ESEP. So, to maximize the stable region, the network lifetime and reliability of WSN system the concept of heterogeneity can be applied to WSN system.

In the design of clustering protocol for wireless sensor network, the main problems that require immediate attention are described as follows:

- How to effectively and efficiently arranged the various nodes in the heterogeneous network in order to minimize the energy consumptionof nodes

- How to stabilize the energy dissipation of sensor nodes so as to proliferate the stableregion of the network and hence the reliability of network.

\section{Mathematical Model}

Many research work proposed in the past have used the radio dissipation model defined in [5]. Energy is lost in sending and receiving the packets and the consumption of energy is represented in mathematical way as $\mathrm{d}^{2}$ for small distance (for line of sight communication) and $\mathrm{d}^{4}$ for large distance because of multipath fading propagation. The energy losses in transmitting and receiving a l-bit packet are shown below:

$$
\begin{gathered}
E_{T}= \begin{cases}l * E_{\text {elec }}+l * E_{f s} * d^{2} & \text { if } d<d_{o} \\
l * E_{\text {elec }}+l * E_{m p} * d^{4} & \text { if } d>d_{o}\end{cases} \\
E_{R}=E_{\text {elec }} * l
\end{gathered}
$$

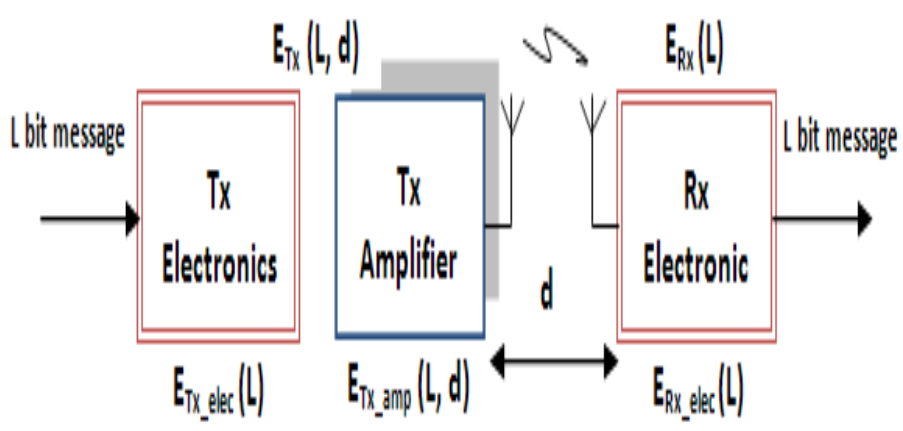

Figure 1. Energy Dissipation Model [5]

where, $E_{\text {elec }}$ is the energy utilized per bit to process transmitter and receiver circuit. $E_{f s}$ and $E_{m p}$ are the free space and multi path losses based on the distance of transmission. If the distance $\mathrm{d}$ is less than threshold $d_{o}$ then free space model is used else multipath model is used. The value of

$$
\mathrm{d}_{o}=\sqrt{\frac{E f}{E_{m p}}} .
$$

The equation 1 and 2 gives the value of energy consumed in transmitting and receiving process respectively. In sending packets, the energy consumed depends on the size of packet and the transmission distance, while in receiving energy lost depends only on the size of data packets received. 


\section{Performance Parameter}

The performance of the clustering protocols is measured on the basis of following criteria's [8]:

- Stability Period: It is the time duration from the beginning of network process until the first sensor node death. Also called as "stable region."

- Instability Period: It is the time duration from the first node death (FND) to death of the last sensor node (LND). Also called as "unstable region."

- Network remaining energy: It gives the value of remaining energy of the whole network and is calculated after each round of transmission of the protocol.

- Network lifetime: It is the time interval from the start of operation (of the sensor network) until the death of the last alive node.

- Number of node alive per round: This instantaneous measure reflects the total number of nodes and that of each type that have not yet expended all of their energy

- Reliability:Reliability is also an important parameter described in [8] and it is noted that the smaller the unstable region, better is the reliability of system.

- Throughput: The throughput of a system is the total number of packets sent from the nodes to cluster head and from cluster heads to base station.

The stability as discussed in [8] is an important parameter of the WSN system. Generally, there is a tradeoff between the reliability and lifetime of system. The last node before dying can still provide some data but there will be no reliability of it.

\section{Proposed Work}

We present an advance deterministic energy proficient clustering algorithm which is extending the work of Deterministic energy efficient algorithm clustering algorithm (DEEC) [1] by implementing the concept of energy heterogeneity. In this technique we have considered four-level clustering keeping the total energy of the system as constant i.e., 102.5 J. We have included an additional node known as superior node in the system to provide the multi-node variety. This modification improves the stability region of the network system and network lifetime as well.

The aim of ADEC protocol is to increase the network lifetime and stability of the network in the presence of node heterogeneity. It is evident that cluster heads utilize more power than other cluster members as they perform multiple functions like receiving and sensing data from cluster members and sending aggregated data to other sensor node or to base station. The cluster head role needs to be rotated among cluster members to make efficient utilization of the resources of network. So ADEC works in rounds like DEC. 


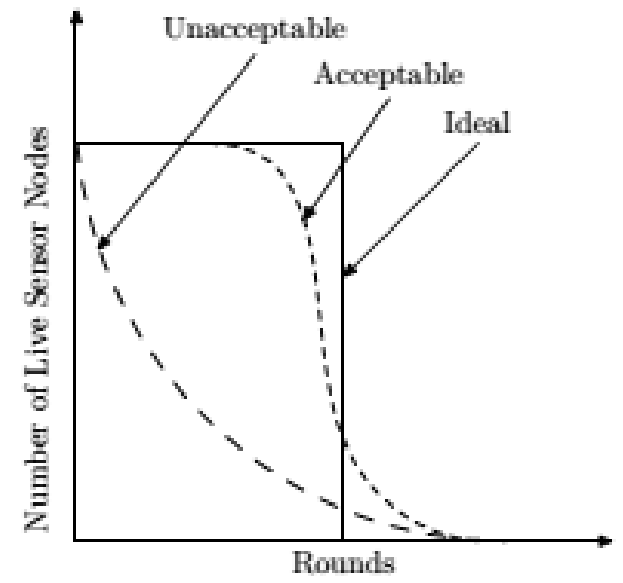

Figure 2. Behaviour of Node Energy Consumption Overtime [19]

\subsection{Network Model}

The network is made of $\mathrm{n}$ nodes in a network area of $100 * 100$. Four types of sensor nodes are considered namely normal, intermediate, advance and superior [17, 18]. Let, $\mathrm{m}$, $\mathrm{x}$ and $\mathrm{v}$ be the fraction of nodes that are advance, intermediate and superior respectively. Superior nodes possess $(1+c)$ times, advance nodes $(1+b)$ times and intermediate nodes $(1+a)$ times more energy than normal nodes. $E_{0}$ is the initial energy of each normal node. Number of normal nodes is, $n=(1-m-X-v)$

For a four-level heterogeneous WSN the overall initial energy of system is given by: $\mathrm{E}_{\mathrm{tot}}=\mathrm{n}^{*} \mathrm{E}_{\mathrm{o}}+(1+\mathrm{a}) * \mathrm{~m} * \mathrm{E}_{\mathrm{o}}+(1+\mathrm{b}) * \mathrm{x}^{*} \mathrm{E}_{\mathrm{o}}+(1+\mathrm{c}) * \mathrm{E}_{\mathrm{o}}(3)$

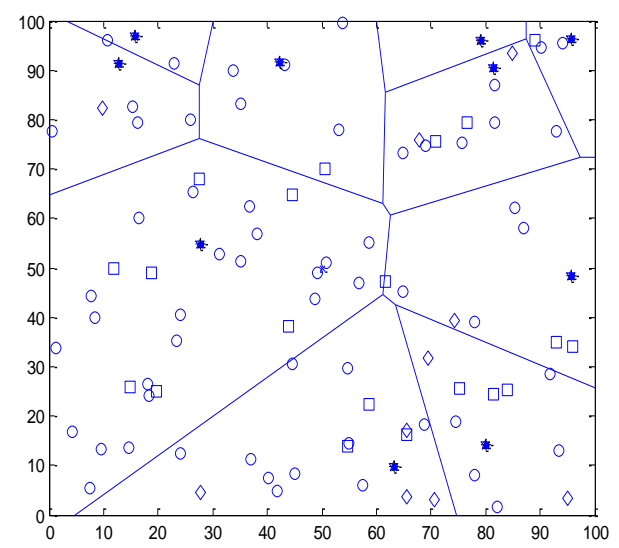

Figure 3. Initial View of Clustered Network in ADEC

Figure 3 is a snapshot of the system when network is initialized showing different clustered region. Four types of nodes normal, intermediate, advanced and super are shown with different symbols.

\subsection{Network Assumptions}

Some assumptions are made for the sensor nodes as well as for the network as follows:

- There is a uniform random deployment of the sensor nodes in the network

- The base station is place at the center of the WSN

- All nodes are equipped with equal capabilities of processing and communication 
- There is four-level heterogeneity in terms of energy in the nodes; some nodes are equipped with more energy than others. Four nodes are taken with different energy level.

\subsection{The ADEC PROTOCOL}

In this part we will present the details of our ADEC protocol. ADEC implements the same concept as in DEC [1], in terms of selecting the cluster head $(\mathrm{CH})$ which is based solely on the residual energy of the nodes. DEC is proposed for both homogeneous and heterogeneous system. But in heterogeneous setting of DEC three level node heterogeneity is taken and three types of nodes are present in it; normal, intermediate and advance nodes. ADEC extends the heterogeneity of system to four-level energy heterogeneity and four types of nodes are taken; normal nodes, intermediate nodes, advance nodes and superior nodes. The superior node has the greater energy than the advanced nodes. In terms of mathematics, the energy level of the nodes can be expressed as $\mathrm{E}_{\text {nor }}<\mathrm{E}_{\text {inter }}<\mathrm{E}_{\text {advan }}<\mathrm{E}_{\text {sup. }}$.

The set up phase of ADEC is same as in DEC. The energy of nodes is known prior the network operation, so the cluster head $(\mathrm{CH})$ election process is arranged in such a way to use only the residual energy of each node. Like in DEC, BS initiates the cluster head election process by electing $\mathrm{N}_{\mathrm{opt}}$ cluster heads at round $\mathrm{m}=1$. After that in each subsequent round the election of cluster head is done on the basis of the residual energy (RE) of the nodes. Each selected cluster head announce their part by broadcasting a join-request message containing the ID-CH (Cluster head ID), ID-CM (Cluster member ID), RE-CM (residual energy of cluster member). The RE data of $\mathrm{CM}$ is known by the associated $\mathrm{CH}$ of that cluster. The current $\mathrm{CH}$ checks the RE-CM data and decides to whether continue as the $\mathrm{CH}$ or renounce its duty as cluster head and select another node with high value of RE to be cluster head. After the end of set up phase, the transmission phase begins the. In figure 4 the network operations of ADEC are described. Three stages are shown, network initialization, set up phase and steady state phase.

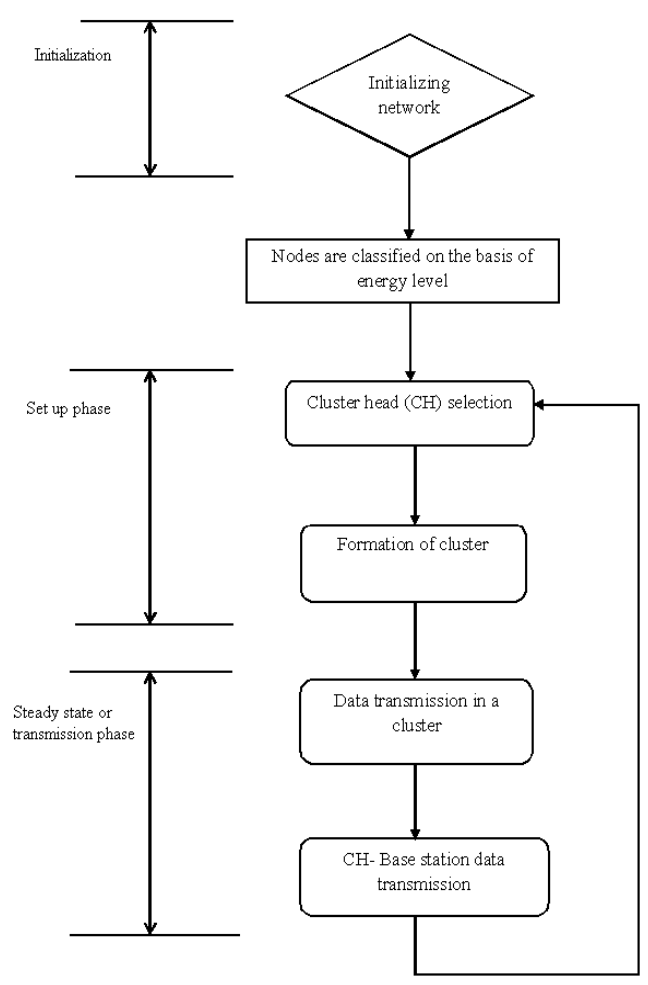

Figure 4. Flow Chart of Network Operations 


\section{Simulation and Result Discussion}

In this, we have implemented the ADEC in MATLAB 2012b, examined the performance of WSN at four level node hierarchies and compare the results with existing clustering protocol DEC, and LEACH (homogeneous setting). The parameters taken in the simulation are described in Table 1 . The base station is located at $(50,50)$.

Table 1. Parameters of Simulation

\begin{tabular}{|l|l|}
\hline Parameter & Values \\
\hline Network area & $100 * 100$ \\
\hline Number of nodes (n) & 100 \\
\hline$E_{o}$ & $0.5 \mathrm{~J}$ \\
\hline K (packet size) & 4000 \\
\hline Number of rounds & 6000 \\
\hline$P_{\text {opt }}$ & 0.1 \\
\hline$E_{\mathrm{fs}}$ & $10 \mathrm{pJ} / \mathrm{bit}^{\mathrm{m}} \mathrm{m}^{2}$ \\
\hline $\mathrm{E}_{\mathrm{mp}}$ & $0.0013 \mathrm{pJ} / \mathrm{bit} / \mathrm{m}^{4}$ \\
\hline $\mathrm{E}_{\text {elec }}$ & $50 \mathrm{nj} / \mathrm{bit}$ \\
\hline $\mathrm{E}_{\mathrm{DA}}$ & $5 \mathrm{nj} / \mathrm{bit} / \mathrm{message}$ \\
\hline
\end{tabular}

Four-level energy heterogeneity: In this we have considered, $10 \%$ nodes are advanced equipped with $2 \mathrm{~J}, 20 \%$ nodes are intermediate with $1.25 \mathrm{~J}, 10 \%$ nodes are superior nodes with $2.75 \mathrm{~J}$ energy and remaining $60 \%$ are normal nodes with $0.5 \mathrm{~J}$ energy. The total energy of system is $102.5 \mathrm{~J}$ which is kept same for both DEC and ADEC for fairness.

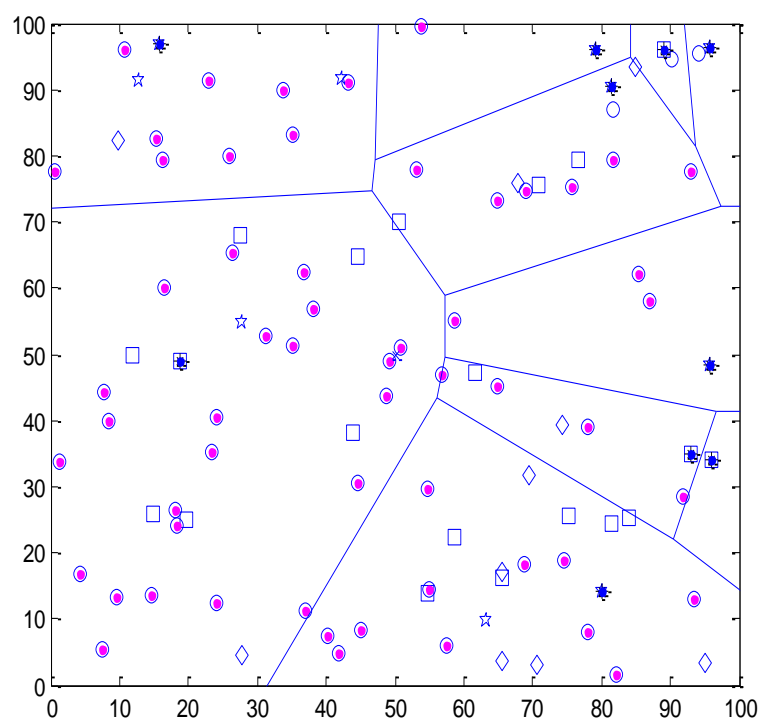

Figure 5. View of Network when some Nodes are Half Dead 


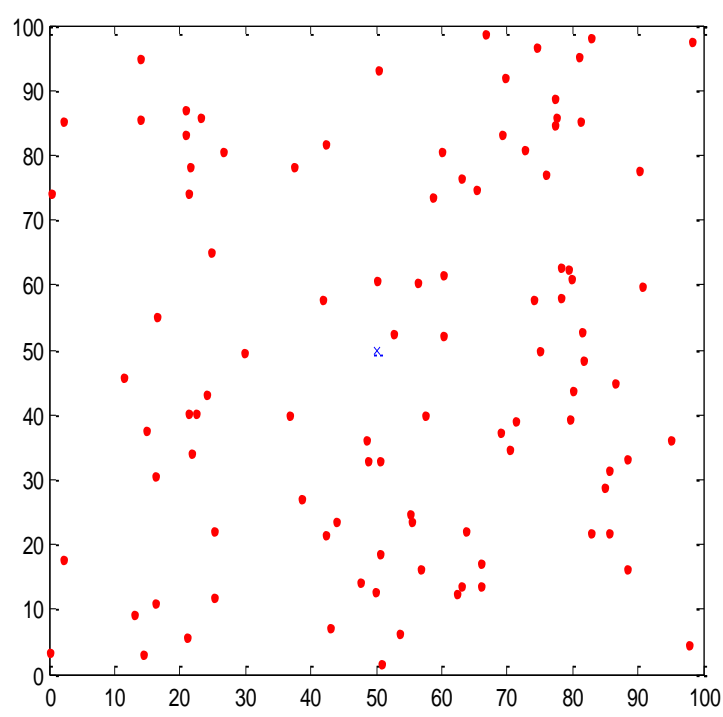

\section{Figure 6. View of Network when all Nodes are Dead}

Figure 5 gives the view of the network when some nodes are half dead means that the energy of the nodes becomes half of the initial value. The half dead nodes are shown with pink color. The Figure 6 is an instance of network when all nodes are dead, shown with red color.

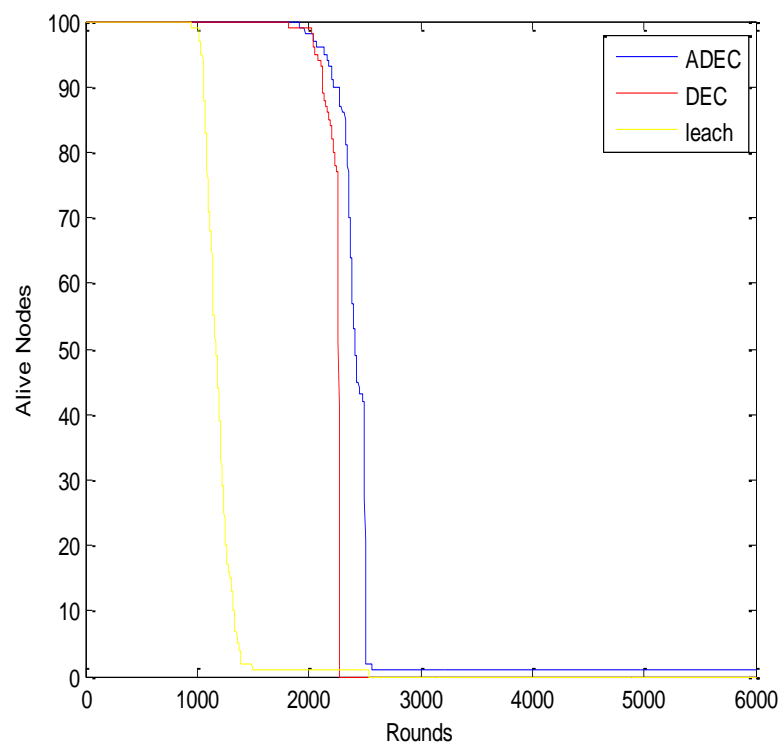

Figure 7 Number of Nodes Alive over each Round 


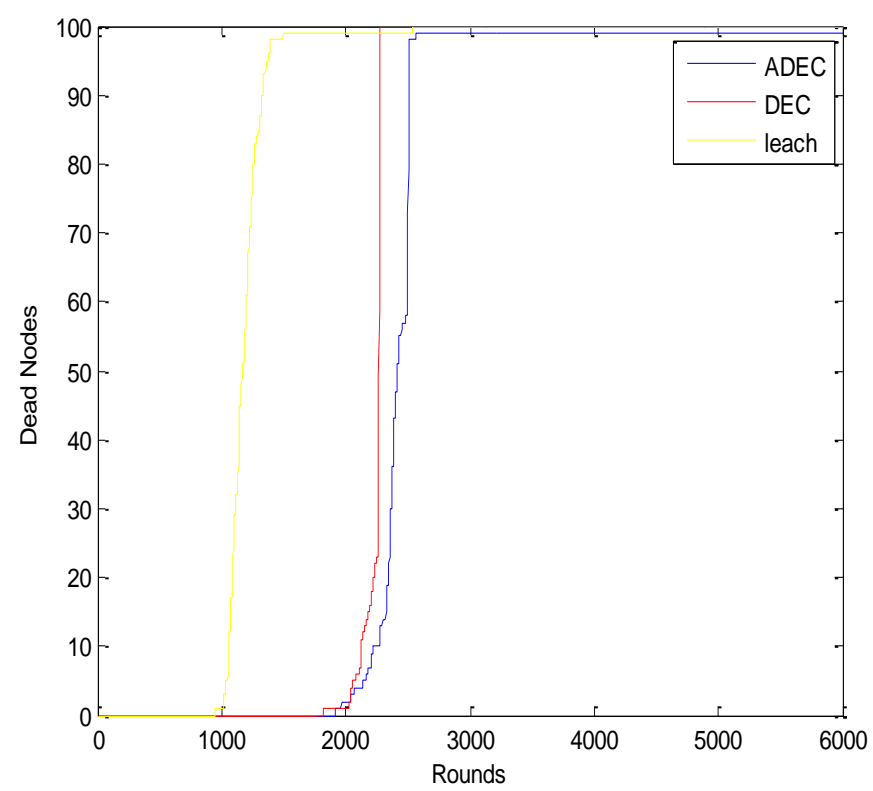

Figure 8. Number of Dead Nodes over each Round

Figure 7 and 8 shows respectively the number of alive nodes and number of dead during the networklifetime after each round for ADEC, DEC, and LEACH. It clearly shows that by increasing the heterogeneity lifetime of the system increases. The corresponding values of FND, LND are also improved. Table 2 gives the details of FND and LND for LEACH, DEC and ADEC respectively. From Figure 7 and 8we can conclude that after the inclusion of superior nodes i.e., increasing the heterogeneity of the network the stable region of the network is increased. The instable period is also less. The network lifetime is also increased in ADEC as compared to DEC.

Table 2. FND and LND for DEC and ADEC

\begin{tabular}{|l|l|l|}
\hline $\begin{array}{l}\text { Clustering } \\
\text { protocols }\end{array}$ & FND & LND \\
\hline DEC & 1816 & 2273 \\
\hline ADEC & 1922 & 5982 \\
\hline LEACH & 949 & 2537 \\
\hline
\end{tabular}




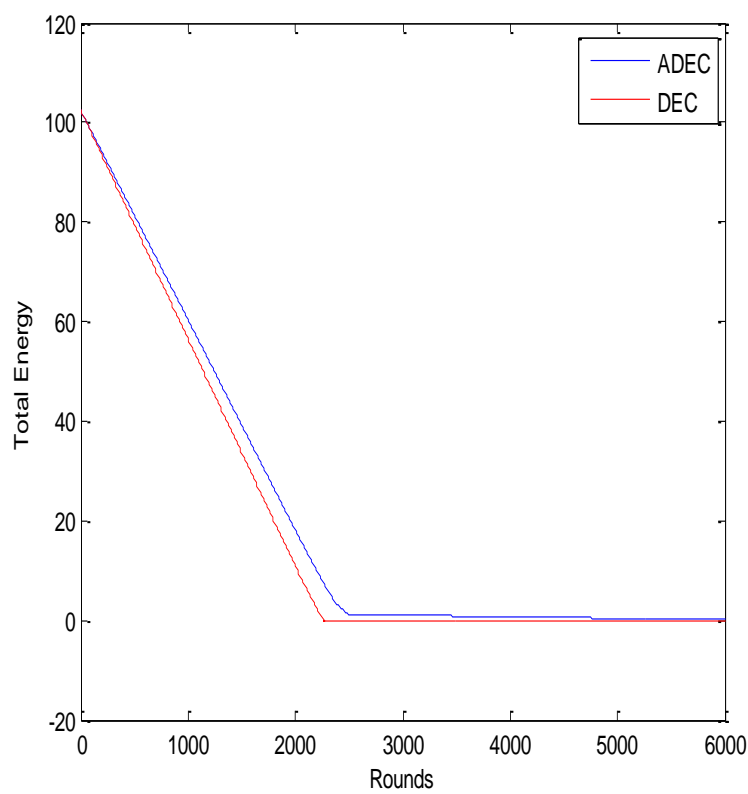

Figure 9. Total Energy (in Joule) of DEC and ADEC after each Round

Figure 9 shows the total energy of system after each round for DEC and ADEC. The initial energy of the system is kept same in both the algorithm for fairness. We can see from the graph that the energy dissipated each round is less in in ADEC then DEC.

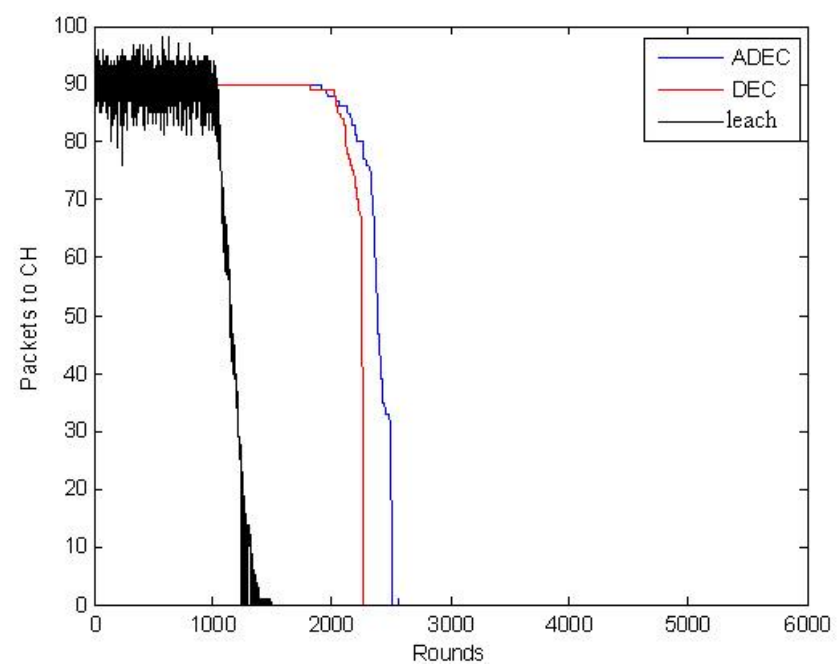

Figure 10. Packets Sent to $\mathrm{CH}$ over each Round 


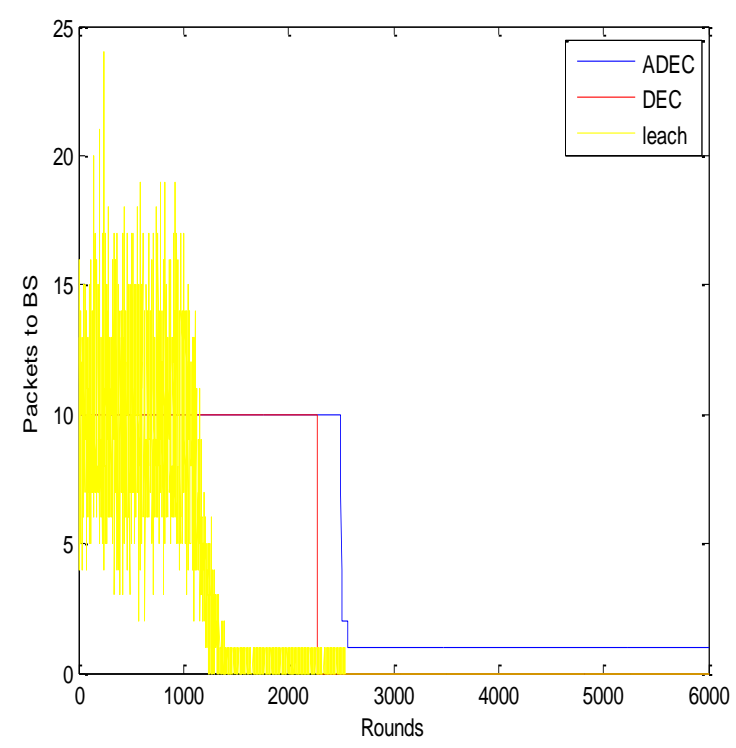

Figure 11. Packets Sent to BS

In Figure 10 and 11, the packets sent to $\mathrm{CH}$ and $\mathrm{BS}$ is shown for each round. From the graph we can conclude that the number of packets sent for ADEC is more as compared to other algorithms. Hence it can also be concluded that the throughput of ADEC is more than DEC.

\section{Conclusion}

In this paper we have given an improved clustering algorithm in the presence of node heterogeneity. Proposed ADEC is an energy efficient clustering algorithm for heterogeneous WSNs, with inclusion of four types of nodes with different energy level keeping the total energy of the system as same in the DEC algorithm. The proposed algorithm is implementing the same concept for cluster formation and cluster head selection as in DEC with increased system heterogeneity. Cluster head selection is based on the residual energy of the system, so nodes with high energy level have high chances of being selected as the cluster head. The node heterogeneity significantly improved the stable region of the network from the previous algorithms such as LEACH and DEC. in addition to the stability of the network, the number of live nodes is also comparatively higher than previous algorithms before the network turns unreliable to utilize. The FND and LND for DEC and ADEC are 1816, 2273 and 1922, 5982 respectively. The energy dissipation per round for ADEC is also less as compared to DEC. Instability period is also less which makes the protocol reliable. Like DEC, in ADEC there are no uncertainties involved in the cluster head selection process which evenly distribute energy load among sensor nodes. The throughput of ADEC is also higher than DEC. ADEC is an efficient protocol as compared to the LEACH and DEC in terms of greater stability period, network lifetime, less total dissipation energy of the system and higher throughput.

\section{References}

[1] F. A. Aderohunmu, J. D. Deng and M. K. Purvis, "A deterministicenergy-efficient clustering protocol for wireless sensor networks", Proceedings of Seventh IEEE International Conference on Intelligent Sensors, Sensor Networks and Information Processing, (2011), pp. 341-346.

[2] F. L. Lewis, D. J. Cook and S. K. Das, "Wireless Sensor Networks Smart Environments: Technologies", Protocols, and Applications John Wiley, New York, (2004).

[3] F. A. Aderohunmu, "Energy Management Techniques in Wireless Sensor Networks: Protocol Design and Evaluation”, M.S. Thesis, Dept. Information Science, Otago University, New Zealand, (2010). 
[4] M. Haase and D. Timmermann, "Low energy adaptive clustering hierarchy with deterministic clusterhead selection", In IEEE Conference on Mobile and Wireless Communications Networks (MWCN), (2002), pp. 368-372.

[5] W. R. Heinzelman, A. Chandrakasan and H. Balakrishnan, "An Application-Specific Protocol Architectures for Wireless Networks", IEEE Transactions on Wireless Communications, vol. 1, (2002), pp. 660-670.

[6] L. Qing, Q. Zhu, and M. Wang, "Design of a distributed energy-efficient clustering algorithm for heterogeneous wireless sensor networks", Computer Communication, vol. 29, (2006), pp. 2230-2237.

[7] I. F. Akyildiz, W. Su, Y. Sankarasubramaniam and E. Cayirci, "A survey on sensor networks", IEEE Communications Magazine, vol. 40, (2002), pp. 102-114.

[8] G. Smaragdakis, I. Matta and A. Bestavros, "SEP: A Stable Election Protocol for clustered heterogeneous wireless sensor networks", Proceeding of the International Workshop on SANPA, (2004).

[9] J. Ibriq and I. Mahgoub, "Cluster-Based Routing in Wireless Sensor Networks: Issues and Challenges", Proceeding of the International Symposium on Performance Evaluation of Computer and Telecommunications System, San Jose, California, USA, (2004), pp. 759-766.

[10] C. S. R. Murthy and B. S Manoj, "Ad Hoc Wireless Networks Architectures and Protocols", Prentice Hall Communication Engineering and Emerging Technologies Series, (2004).

[11] N. Jamal, A. Karaki and A. E. Kamal, "RoutingTechniques in Wireless Sensor Networks: A Survey", IEEE Wireless Communications, vol. 11, iss. 6, (2004), pp. 26-28.

[12] O. Younis and S. Fahmy, "HEED: A Hybrid, EnergyEfficient, Distributed Clustering Approach for Ad Hoc Sensor Networks", IEEE Trans. Mobile Computing, vol. 3, no. 4, (2004), pp. 366-379.

[13] M. Bala and L. Awasthi, "Proficient D-SEP Protocol with Heterogeneity for Maximizing the Lifetime of Wireless Sensor Networks", International Journal of Intelligent System and Applications, vol. 7, (2012), pp. $1-15$.

[14] F. A. Aderohunmu, J. D. Deng and M. K. Purvis, "Enhancing Clustering in Wireless Sensor Networks with Energy Heterogeneity", International Journal of Business Data Communications and Networking(Accepted, to appear in the forthcoming issue, (2011).

[15] K. Latif, M. Jaffar, N. Javaid, M. N. Saqib, U. Qasim and Z. A. Khan, "Performance Analysis of Hierarchical Routing Protocols in Wireless Sensor Networks", 5th International Workshop on Next Generation of Wireless and Mobile Networks (NGWMN 2012) in conjunction with 7th IEEE International Conference on Broadband and Wireless Computing, Communication and Applications (BWCCA 2012), Victoria, Canada, (2012).

[16] W. R. Heinzelman, A. P. Chandrakasan and H. Balakrishnan, "Energy efficient communication protocol for wireless micro sensornetworks", Proceedings of the 33rd Hawaii International Conference on System Sciences (HICSS-33), (2000).

[17] Y. Mao, Z. Liu, L. Zhang and X. Li, "An Effective Data Gathering Scheme in Heterogeneous Energy Wireless Sensor Networks", International Conference on Computational Science and Engineering, (2009).

[18] D. Kumar, T. C. Aseri and R. B. Patel, "EEHC: Energy efficient heterogeneous clustered scheme for wireless sensor networks", ELSEVIER, Compute Communications, vol. 32, (2009), pp. 662-667.

[19] S. Gamwarige and C. Kulasekere, "An algorithm for energy driven cluster head rotation in a distributed wireless sensor network", Proceeding of the International Conference on Information and Automation, (2005), pp. 354-359. 